\title{
Clinicopathological features of the superficial spreading type of early gastric cancer
}

\author{
Yuichi Kasakura, Masashi Fuji, Fumiro Mochizuki, Shigeru Imai, Noriaki Kanamori, and Tetsuro Suzuki \\ Third Department of Surgery, Nihon University School of Medicine, 30-1 Oyaguchi-kamimachi, Itabashi-ku, Tokyo 173-8610, Japan
}

\begin{abstract}
:
Background. During a 10-year period (1986-1995), 59 of 538 patients with early gastric cancer $(11.0 \%)$ had the superficial spreading type of gastric cancer. We attempted to elucidate the clinicopathological features and investigated the influence of those features on surgical procedures and patient prognoses.

Methods. These 59 superficial spreading lesions were analyzed with respect to macroscopic type, lymph node (LN) metastasis, recurrent pattern, and method of surgical operation. In addition, the lesions were compared with those of 393 other patients with small-sized cancer.

Results. In both groups, the IIc type macroscopic lesion occurred most frequently, and the depressed subtype occurred more frequently than the elevated subtype. There was no significant histologic difference between the groups. The incidence of $\mathrm{LN}$ metastasis was $8.7 \%$ in early gastric cancer, $7.1 \%$ in small-sized cancer, and $20.3 \%$ in superficial spreading cancer. The incidence of lymphovascular invasion was $24.4 \%$ in small-sized cancer and $\mathbf{5 0 . 8} \%$ in superficial spreading cancer. The incidences of $\mathrm{LN}$ metastasis and lymphovascular invasion were greater in superficial spreading cancer than in smallsized cancer. Despite extensive preoperative examination, determination of the tumor margin was impossible in 26 of the 59 patients with superficial spreading cancer. The incidence of recurrence was $2.0 \%$ in small-sized cancer and $5.1 \%$ in superficial spreading cancer.

Conclusions. A wide resection with extensive lymph node dissection (D2 or more) seems to be an appropriate treatment for the superficial spreading type of early gastric cancer.
\end{abstract}

Key words: superficial spreading cancer, early gastric cancer, clinicopathological features, recurrence pattern, surgical operation

Reprint requests to: Y. Kasakura

Received for publication on Sept. 17, 1998; accepted on July 21, 1999

\section{Introduction}

Malignant cells of early gastric cancer extend through the muscularis mucosa into the submucosa, but do not deeply penetrate the muscularis propria. Early gastric cancer now accounts for over $40 \%$ of all gastric cancer cases in most Japanese hospitals. This large number of patients with early gastric cancer has allowed the investigation of an unfamiliar disease for which minimal surgery is not yet indicated: the superficial spreading type of early gastric cancer. Approximately $10 \%$ of patients with early gastric cancer have the superficial spreading morphology [1-3]. It has often been reported that superficial spreading cancer is defined as that with lesions more than $25 \mathrm{~cm}^{2}(5 \times 5 \mathrm{~cm})$ in size. The treatment results for early gastric cancer in Japan are satisfactory: the incidence of lymph node (LN) metastasis is $15 \%-20 \%$ [4-6], and the recurrence rate is approximately $2 \%$. In these reports, the incidences of LN metastasis $(10 \%-20 \%)$ and recurrence $(2 \%-4 \%)$ include those of superficial spreading cancer [6-14]. Recently, endoscopic mucosal resection, modified surgery, and laparoscopic wedge resection have been performed in early gastric cancer; however, the endoscopic and modified surgical procedures have not been successful in eliminating superficial spreading cancer $[15,16]$. We attempted to elucidate the clinicopathological features of patients with the superficial spreading type of early gastric cancer by comparing these features with those in small-sized early gastric cancer. We also investigated the influence of these features on surgical procedures and patient prognoses.

\section{Patients and methods}

\section{Patients}

We investigated the medical records of 1411 patients with gastric cancer who had undergone surgical treat- 
ment at the Third Department of Surgery of Nihon University School of Medicine during the 10-year period from 1986 to 1995 . Early gastric cancer was found in 538 patients $(38.1 \%)$; there was intramural carcinoma in 244 patients $(45.4 \%)$ and submucosal carcinoma in 294 patients $(54.6 \%)$. "Superficial spreading" lesions were defined as cancer lesions of $25 \mathrm{~cm}^{2}(5 \times 5 \mathrm{~cm})$ or greater in size. "Small-sized" lesions were defined as cancer lesions of $2 \times 2 \mathrm{~cm}$ or smaller in size. Of the 538 patients with early gastric cancer, 59 (11.0\%) had "superficial spreading" lesions, and 40 of these $(67.8 \%)$ had submucosal carcinoma. Small-sized cancer occurred in 393 patients $(73.0 \%)$, including 216 patients $(55.0 \%)$ with submucosal carcinoma.

\section{Methods}

The clinicopathological features of the 59 patients with superficial spreading lesions of early gastric cancer were compared with those of the 393 patients with smallsized cancers. Early gastric cancer was classified as macroscopic type 0 (superficial, flat tumors with or without minimal elevation or depression), according to the usual gastric cancer typing. Macroscopic type 0 was subdivided as follows: I, protruded; IIa, superficial elevated; IIb, flat; IIc, superficial depressed; and III, excavated. The superficial spreading and small-sized types of cancer were divided into two subtypes based on the macroscopic appearance of cancer: (1) elevated subtype (I, IIa, IIa + X) and (2) depressed subtype (IIb, $\mathrm{IIb}+\mathrm{X}$, IIc, IIc $+\mathrm{X})$. Histopathological classifications were based on the general rules specified in "Japanese Classification of Gastric Cancer," published by the Japanese Research Society for Gastric Cancer [17]. These features were analyzed statistically using the $\chi^{2}$ test.

\section{Results}

The clinicopathological features of superficial spreading lesions were compared with those of small-sized early gastric cancer. Details are shown in Tables 1 to 5.

Table 1. Clinicopathological findings for superficial spreading type and small-sized type

\begin{tabular}{|c|c|c|c|}
\hline Variable & $\begin{array}{c}\text { Superficial spreading } \\
\text { type }(\%)\end{array}$ & $\begin{array}{l}\text { Small-sized } \\
\text { type }(\%)\end{array}$ & $P$ value \\
\hline Number of cases & 59 & 393 & \\
\hline $\begin{array}{l}\text { Macroscopic appearance } \\
\text { I } \\
\text { I }+ \text { X } \\
\text { IIa } \\
\text { IIa }+ \text { X } \\
\text { IIb } \\
\text { IIb }+ \text { X } \\
\text { IIc } \\
\text { IIc }+ \text { X } \\
\text { III } \\
\text { III }+ \text { X }\end{array}$ & $\begin{array}{c}4 \\
5 \\
4 \\
5 \\
0 \\
3 \\
29(49.2) \\
9 \\
0 \\
0\end{array}$ & $\begin{array}{c}19 \\
3 \\
26 \\
17 \\
6 \\
3 \\
265(67.4) \\
38 \\
1 \\
15\end{array}$ & $<0.0001$ \\
\hline $\begin{array}{l}\text { Macroscopic subtype } \\
\text { Elevated } \\
\text { Depressed }\end{array}$ & $\begin{array}{c}17 \\
42(71.2)\end{array}$ & $\begin{array}{cl}65 \\
328 & (83.5)\end{array}$ & $<0.01$ \\
\hline $\begin{array}{l}\text { Lymph node dissection }^{\mathrm{a}} \\
\text { D1 } \\
\text { D2 or more }\end{array}$ & $\begin{array}{c}19 \\
40(67.8)\end{array}$ & $\begin{array}{c}232 \\
161(41.0)\end{array}$ & $<0.0001$ \\
\hline $\begin{array}{l}\text { Curability } \\
\text { A } \\
\text { B } \\
\text { C }\end{array}$ & $\begin{array}{c}45(76.3) \\
12 \\
2\end{array}$ & $\begin{array}{c}377(95.9) \\
11 \\
5\end{array}$ & $<0.0001$ \\
\hline
\end{tabular}

I, protruded type; IIa, superficial elevated type: IIb, flat type; IIc, superficial depressed type: III, excavated type; X, mixed type. Eievated type, I + IIa + (IIa + X); depressed type; IIb + (IIb + $\mathrm{X})+\mathrm{IIc}+(\mathrm{IIc}+\mathrm{X})$. DI, dissection of group 1 lymph nodes; D2, dissection of group 2 lymph nodes, Curability A, no residual tumor and a high probability of cure; Curability B, no residual tumor, but not definable as curability A; Curability C, definite residual rumor

${ }^{a}$ See text for explanation of D1 and D2 
Table 2. Histological findings for superficial spreading type and small-sized type

\begin{tabular}{lccc}
\hline Variable & $\begin{array}{c}\text { Superficial spreading } \\
\text { type }(\%)\end{array}$ & $\begin{array}{c}\text { Small-sized } \\
\text { type }(\%)\end{array}$ & $P$ value \\
\hline Depth of invasion & 19 & 177 & $<0.05$ \\
$\quad$ Mucosa (m) & $40(67.8)$ & $216(55.0)$ & \\
Submucosa (sm) & 2 & 5 & NS \\
Histological typing & 9 & 106 & \\
Pap & 15 & $123(31.3)$ & \\
Tub 1 & $23(39.0)$ & 118 & \\
Tub 2 & 10 & 40 & $<0.005$ \\
Por & 0 & 1 & \\
Sig & & $28(7.1)$ & \\
Muc & $12(20.3)$ & 365 & \\
Lymph node metastasis & 53 & & \\
Positive & & $96(24.4)$ & \\
Negative & $30(50.8)$ & 297 & \\
Lymphovascular invasion & 29 & & \\
Positive & & & \\
Negative & & & \\
\hline
\end{tabular}

pap, papillary adenocarcinoma; tub 1, well differentiated adenocarcinoma; tub 2, moderately differentiated adenocarcinoma; por, poorly differentiated adenocarcinoma; sig, signal-ring cell carcinoma; muc, mucinous adenocarcinoma; NS, not significant

Table 3. Histological type and macroscopic subtypes

\begin{tabular}{lcccccc}
\hline & \multicolumn{2}{c}{$\begin{array}{c}\text { Superficial spreading } \\
\text { type }(\%)\end{array}$} & & Small-sized type (\%) & \\
\cline { 2 - 3 } $\begin{array}{l}\text { Variable } \\
\text { Subtype }\end{array}$ & Elevated & Depressed & & Elevated & Depressed & $P$ value \\
\hline Histological type & & & & & & $<0.0001$ \\
Pap & 1 & & & 2 & 0 & \\
Tub 1 & 2 & $7(41.2)$ & & $36(55.4)$ & 69 & \\
Tub 2 & 12 & 3 & & 18 & 106 & \\
Por & $19(45.3)$ & 4 & & 9 & $110(33.5)$ & \\
Sig & 8 & 2 & & 0 & 43 & \\
Muc & 0 & 0 & & 0 & 0 &
\end{tabular}

\section{Macroscopic characteristics}

Type IIc occurred more frequently than any other macroscopic type in both the small-sized cancer group and the superficial spreading cancer group. It occurred in 29 of the 59 patients with superficial spreading cancer $(49.2 \%)$ and in 265 of the 393 patients with small-sized cancer $(67.4 \%)$. Type IIc $+\mathrm{X}$ (mixed type) was the second most frequent macroscopic type in both groups. As for macroscopic subtypes, the incidence of the depressed subtype was higher than that of the elevated subtype in both groups (Table 1).

\section{Histologic characteristics}

Submucosal invasion was more prominent in superficial spreading lesions than in small-sized cancer lesions (Table 2). In both groups, there was no significant difference in the incidence among well differentiated ad- enocarcinoma (tub 1), moderately differentiated adenocarcinoma (tub 2), and poorly differentiated adenocarcinoma (por). In superficial spreading cancer, por occurred most frequently, followed by tub 2. In both groups, the incidence of tub 1 was higher in the depressed subtype than in the elevated subtype. In superficial spreading cancer, the incidences of tub 2 and por were higher in the elevated subtype than in the depressed subtypes. In contrast, in small-sized cancer, the incidences of tub 2 and por were higher in the depressed subtype than in the elevated subtype. Although there was no significant difference in the histological characteristics of the macroscopic subtypes (elevated and depressed) among the small-sized cancer lesions, in the superficial spreading cancer, the histological type of the depressed subtype was less differentiated than that of the elevated subtype (Table $3)$. 
Table 4. Characteristics of lymph node metastasis

\begin{tabular}{lccc}
\hline Variable & $\begin{array}{c}\text { Superficial spreading } \\
\text { type }(\%)\end{array}$ & $\begin{array}{c}\text { Small-sized } \\
\text { type }(\%)\end{array}$ & $P$ value \\
\hline Number of cases & $12(20.3)$ & $28(7.1)$ & $<0.005$ \\
Depth of invasion & 0 & $2(1.1)$ & $\mathrm{NS}$ \\
m & $12(30.0)$ & $26(12.0)$ & \\
sm & & & $\mathrm{NS}$ \\
Macroscopic appearance & $3(75.0)$ & $3(15.8)$ & \\
I & $1(25.0)$ & $2(7.7)$ & \\
IIa & 0 & $3(17.6)$ & \\
IIa + X & 0 & $1(5.9)$ & \\
IIb & $1(33.4)$ & $1(16.7)$ & \\
IIb + X & $6(20.7)$ & $14(5.3)$ & \\
IIc & $1(11.1)$ & $4(10.5)$ & \\
IIc + X & & $8(12.3)$ & \\
Macroscopic subtype & $4(22.2)$ & $20(6.1)$ & \\
Elevated & $8(19.5)$ & & \\
Depressed & & 0 & \\
Histological type & 0 & $3(10.7)$ & \\
Pap & $1(8.3)$ & $14(53.8)$ & \\
Tub 1 & $3(25.0)$ & $10(35.7)$ & \\
Tub 2 & $6(50.0)$ & $1(3.8)$ & \\
Por & $2(16.7)$ & & \\
Sig & & & \\
\hline
\end{tabular}

Table 5. Recurrence of superficial spreading type and small-sized type

\begin{tabular}{|c|c|c|c|}
\hline Variable & $\begin{array}{l}\text { Superficial spreading type } \\
(\%)\end{array}$ & $\begin{array}{l}\text { Small-sized type } \\
(\%)\end{array}$ & $P$ value \\
\hline Number of cases & $3(5.1)$ & $8(1.7)$ & \\
\hline Depth of invasion & & & NS \\
\hline $\mathrm{m}$ & 0 & 1 & \\
\hline $\mathrm{sm}$ & 3 & 7 & \\
\hline Lymph node metastasis & & & $<0.05$ \\
\hline Positive & 3 & 2 & \\
\hline Negative & 0 & 6 & \\
\hline Lymphovascular invasion & & & NS \\
\hline Positive & 3 & 6 & \\
\hline Negative & 0 & 2 & \\
\hline Recurrence pattern & & & NS \\
\hline Distant metastasis & 2 & 3 & \\
\hline $\begin{array}{l}\text { Local lesion or lymph } \\
\text { node }\end{array}$ & 0 & 4 & \\
\hline Stomach remnant & 1 & 1 & \\
\hline
\end{tabular}

\section{Lymph node (LN) metastasis (Table 4)}

LN metastasis was found in 47 of the 538 early gastric cancer patients $(8.7 \%)$. Of these, 28 patients $(59.5 \%)$ had small-sized cancer and 12 patients (25.5\%) had superficial spreading cancer. The incidence of LN metastasis was $1.3 \%$ in all the patients with intramucosal carcinoma, $1.1 \%$ in those with small-sized cancer, and $0 \%$ in those with superficial spreading cancer. In the patients with submucosal carcinoma, on the other hand,
LN metastasis was found in $15.0 \%$ of all the patients with early gastric cancer, occurring in $12.0 \%$ of those with small-sized cancer and in $30.0 \%$ of those with superficial spreading cancer. In small-sized cancer, the incidence of LN metastasis was not significantly different among macroscopic types, ranging from $5.3 \%$ to $17.6 \%$. In superficial spreading cancer, however, the incidence of LN metastasis ranged from $11.1 \%$ to $75.0 \%$ among the macroscopic types, with a notable difference. No statistically significant difference in LN metastasis 
was found between superficial spreading cancer and small-sized cancer. Furthermore, the incidence of LN metastasis was not significantly different among the macroscopic types and subtypes, although it was slightly higher in the elevated subtype than in the depressed subtype. In general, the incidence of LN metastasis was higher in the patients with superficial spreading cancer than in those with small-sized cancer. Nevertheless, there was no significant difference between these two groups when they were compared according to both depth of invasion and histological types.

\section{Lymphovascular invasion}

Lymphovascular invasion was observed in 96 of the 393 patients $(24.4 \%)$ (Table 2) with small-sized cancer and in 30 of the 59 patients $(50.8 \%)$ with superficial spreading cancer. The incidence of lymphovascular invasion was $2.2 \%$ in intramucosal carcinoma and $50.5 \%$ in submucosal carcinoma. There was no correlation between LN metastasis and lymphovascular invasion. The incidence of lymphovascular invasion was higher in superficial spreading cancer than in smallsized cancer, as was the case with LN metastasis.

\section{Surgical operation}

Gastrectomy, with D1 (dissecting group $1 \mathrm{LN}$ ) or D2 (dissecting group 1 and group 2 regional LN) lymph node dissection was performed in the 393 patients with small-sized cancer (Table 1). Of these, 161 patients (41.0\%) underwent D2 lymph node dissection. Most patients with superficial spreading lesions underwent total or subtotal gastrectomy with a safe surgical margin. D2 or more extensive lymph node dissection was performed in 40 of the 59 patients with superficial spreading cancer. As the tumor margin could not be determined by various preoperative examinations in 26 of these 59 patients, it was confirmed by intraoperative gastrotomy or by a macroscopic examination of the resected specimens in these patients. These examinations revealed indistinct tumor margins in 20 patients and distinct tumor margins in 6 patients. The 20 patients with indistinct margins underwent intraoperative histological examination. As a result, 12 of these patients $(20 \%$ of the 59 patients with superficial spreading cancer) underwent resection additional to the initial gastrectomy, due to positive surgical margins. Gastric resection of curability A (i.e., no residual tumor and high probability of cure) was performed in 377 patients with small-sized cancer $(95.9 \%)$, and in 45 patients with superficial spreading cancer $(76.3 \%)$.

\section{Typical superficial spreading lesion}

An extensive IIa type of macroscopic lesion with a small IIc type lesion (IIa + IIc) of early gastric carcinoma is shown in Fig. 1. This superficial spreading lesion measured $18.5 \times 17.5 \mathrm{~cm}^{2}$ and was treated by total gastrectomy with D2 lymph node dissection. The histopathological examination revealed that the lesion was intramural carcinoma with tub 1 without lymph node metastasis.

\section{Recurrence}

There was recurrence in 8 of the 393 patients with smallsized cancer $(1.7 \%)$ and in 3 of the 59 patients with superficial spreading cancer (5.1\%) (Table 5). Of the 8 patients with small-sized cancer, 1 had intramucosal carcinoma, and 7 had submucosal carcinoma. All 3 of the patients with recurrent superficial spreading cancer
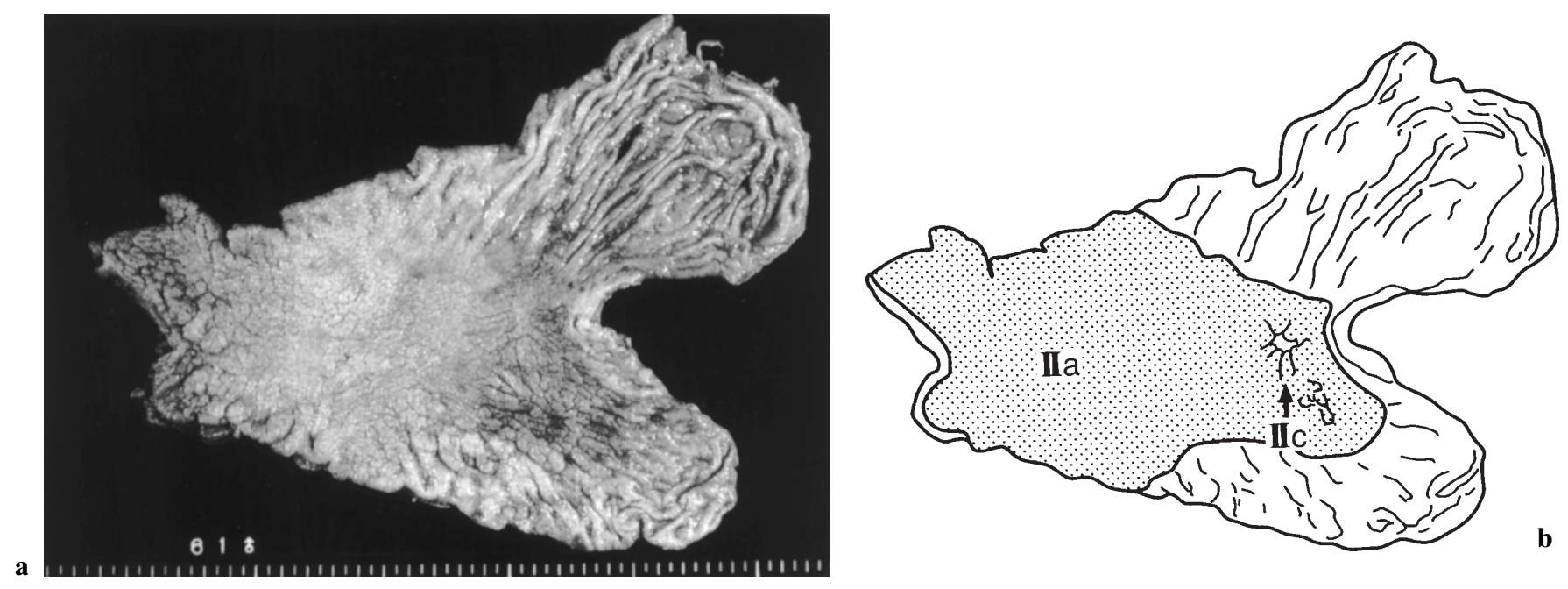

Fig. 1a,b. Typical superficial spreading type; photograph (a) and trace (b) 
had submucosal carcinoma. The recurrence rate was higher in superficial spreading cancer than in smallsized cancer, but the difference was not statistically significant. These recurrence patterns were distant metastases in most patients, but local, LN, and remnant stomach recurrences were found in a few patients. The distant metastases were located in the bones, lungs, liver, and peritoneum. In 6 patients, the recurrence was less than 2 years after surgery, and in 4 patients, more than 5 years after surgery. As for recurrent patterns, there was no difference between small-sized cancer and superficial spreading cancer.

\section{Discussion}

The superficial spreading type of gastric cancer was originally defined by Stout [18]. He identified the wide and superficial spreading activity of the cancer in comparison with its depth of vertical invasion, but did not define its diameter or depth of invasion; it should be noted that his study included advanced cancer. According to Yasui et al. [19], in Japan, the superficial spreading cancer type of early gastric cancer is defined as cancer which is greater than $25 \mathrm{~cm}^{2}(5 \times 5 \mathrm{~cm})$ in size and has characteristics such as diffused appearance, notable histological aggressiveness, and advanced invasion into the lymphatic system. Gastric cancers which have these histological findings generally have poorer prognoses than those without such histological aggressiveness [20]. Thus, the objective of this study was to show that the superficial spreading type of early gastric cancer has a poorer prognosis than that of smallsized cancer. In addition, it has been reported that a great number of recurrence patterns involve distant metastasis to other organs and tissues [7,8,10,21]. These reports suggest the limitations of surgical treatment. In superficial spreading cancer, however, wide resection of the stomach with extensive lymph node dissection has proven useful. Had we performed only limited surgical treatment, cancer remnants and the recurrence of LN metastasis may have increased.

Generally, the incidence of superficial spreading gastric cancer is approximately $10 \%$ of all early gastric cancer cases [1-3]. The macroscopic types of superficial spreading cancer which occur most frequently are types IIc and IIc $+X$ (mixed type). The incidence of the depressed subtype is higher than that of the elevated subtype [22]. It has often been reported that the incidences of LN metastasis and recurrence are higher in superficial spreading cancers than in small-sized cancer [1,23]. Likewise, in our analyses, the incidence of LN metastasis in superficial spreading cancer was $30.0 \%$ (in submucosal carcinoma), which is greater than that found in small-sized cancer. The incidence of lymphovascular invasion was also higher in superficial spreading cancer, which suggests that lymphovascular invasion is also an important factor in the recurrence of early gastric cancer. No correlation was noted, however, between LN metastasis and lymphovascular invasion. In other reports on the macroscopic subtyping, the incidences of LN metastasis and lymphovascular invasion were higher in the elevated subtype than in the depressed subtype [22,24]. Although we also had the same results, the differences were not statistically significant. Although there is no established view as yet which specifically addresses the phenomenon of this difference, it may be due to the nature of the cases studied: e.g., histological type, patterns of growth, and multi centric occurrence $[2,18,25,26]$. In our study, the incidences of recurrence and LN metastasis were higher in superficial spreading cancer than in small-sized cancer. It has been suggested that the risk factors for the recurrence of early gastric cancer are depth (sm), LN metastasis, lymphovascular invasion, and papillary adenocarcinoma [7,8,10,21,27].

As a result of the present study, we suggest that the presence of superficial spreading gastric cancer is an additional risk factor for recurrence. As for the treatment of the superficial spreading type of early gastric cancer, we must first distinguish it from other diseases, namely the polypoid type of malignant lymphoma, multiple polyposis, and chronic gastritis [28-31]. After a diagnosis of the superficial spreading type of early gastric cancer is made, surgeons should understand the nature of the disease before operating. Gastroendoscopic examination is effective in discovering the infiltration area of gastric cancer, but occasionally results in an over- or under- estimation of the infiltration area. Early gastric cancer with a less depressed surface is difficult to diagnose endoscopically, because spreading cancer lesions are difficult to track. To determine the resection line, it is necessary to accurately define the tumor margin; otherwise, all patients must undergo total gastrectomy. Kitamura et al. [23] reported that additional resection was required in $25.0 \%$ of the patients with superficial spreading type of early gastric cancer; Sekiguchi et al. [32] reported the percentage to be $19.9 \%$, and in the present study, this percentage was $20.3 \%$. The tumor margin was so indistinct that the resection line was often difficult to determine. As a result, additional resection was frequently required after distal gastrectomy or subtotal gastrectomy. Given these results, we can now present an excellent method for avoiding unnecessary resection and cancer remnants in the stomach: contrast endoscopy using dye-spraying techniques and endoscopic clipping of the gastric mucosa by biopsy at intervals of $1 \mathrm{~cm}$ [33-35]. However, if determination of the resection line is difficult or if the resected margin is unclear, intraoperative histopatho- 
logical investigation of frozen sections of the surgical margin may be a valid approach.

In conclusion, the superficial spreading types of early gastric cancer should be considered as a disease that is different from other types of early gastric cancer. Its characteristics are invasion almost to the submucosal level, advanced lymph node involvement, and indistinct tumor margin. Wide resection with extensive lymph node dissection (D2 or more) seems to be an appropriate treatment for the disease. Endoscopic mucosal resection, modified reduction surgery, and laparoscopic wedge resection should not be selected for the superficial spreading type of early gastric cancer.

\section{References}

1. Chung Y, Sowa M, Sakurai M. Superficial spreading type of early gastric cancer (in Japanese). Gastroenterol Surg 1994;17:1074-82.

2. Kumagai K, Gonda H, Urabe M, Hayashida Y. Clinicopathological study on superficial spreading type of early carcinoma of the stomach (in Japanese). Jpn J Gastroenterol Surg 1982;15:4538.

3. Hirayama D, Fujimori T, Satonaka K, Nakamura T, Gotoh A, Tokuda Y, et al. The specificity of the penetrating and the superficial spreading types of early gastric cancer. Classification of early gastric cancer using the interactive image analysis system. Dig Endosc 1991;3:16-24.

4. Hirayama K, Fujimori T, Mototsugu A, Maeda S. Clinicopathological and immunohistochemical study on penetrating and superficial spreading type of early gastric cancers. Jpn J Gastroenterol 1990;87:2434-43.

5. Sowa T, Yasuuki K, Masanori N. Surgical approach to early gastric cancer with lymph node metastasis. World J Surg 1991;13:630-66.

6. Maehara Y, Okuyama T, Oshiro T, Baba H, Anai H, Akazawa K, et al. Early carcinoma of the stomach. Surg Gynecol Obstet 1993;177:593-7.

7. Seto $\mathrm{Y}$, Nagawa H, Muto T. Impact of lymph node metastasis on survival with early gastric cancer. World J Surg 1997;21:186-90.

8. Baba $\mathrm{H}$, Maehara $\mathrm{Y}$, Takeuchi $\mathrm{H}$, Inutsuka $\mathrm{S}$, Okuyama $\mathrm{T}$, Adachi et al. Effect of lymph node dissection on the prognosis in patients with node-negative early gastric cancer. Surgery 1995; 117:165-9.

9. Endo M, Habu H. Clinical studies of early gastric cancer. Hepatogastroenterology 1990;37:408-10.

10. Ichiyoshi Y, Toda T, Minamisono Y, Nagasaki S, Yakeishi Y, Sugimachi K. Recurrence in early gastric cancer. Surgery 1990; 107:489-95.

11. Ohta H, Noguchi Y, Takagi K, Nishi M, Kajitani T, Kato Y. Early gastric Carcinoma with special reference to macroscopic classification. Cancer 1987;60:1099-106.

12. Kito T, Yamamura Y, Kobayashi S. Surgical treatment of early gastric cancer. Anticancer Res 1988;8:335-8.

13. Koga S, Kaibara N, Tamura H, Nishidoi H, Kimura O. Cause of late postoperative death in patients with early gastric cancer with special reference to recurrence and the incidence of metachronous primary cancer in other organs. Surgery 1984;6:5116.

14. Kitaoka Y, Yoshikawa K, Hirota T, Itabashi M. Surgical treatment of early gastric cancer. Jpn J Clin Oncol 1984;14:283-93.

15. Sano T, Kobori, Muto T. Lymph node metastasis from early gastric cancer: endoscopic resection of tumor. Br J Surg 1992;79: $241-4$.
16. Imada T, Rino Y, Takahashi M, Suzuki M, Tanaka J, Shinozawa M. Postoperative functional evaluation of pylorus-preserving gastrectomy for early gastric cancer compared with conventional distal gastrectomy. Surgery 1998;123:165-70.

17. Japanese Research Society for Gastric Cancer. Japanese classification of gastric cancer. 1st English ed. Tokyo: Kanehara 1995.

18. Stout AP. Superficial spreading type of carcinoma of the stomach. Arch Surg 1942;44:651-7.

19. Yasui A, Hirase Y, Miyake M, Kidokoro T, Murakami T. Pathology of superficial spreading type of gastric cancer (in Japanese). Stomach and Intestine 1973;8:1305-10.

20. Kodama Y, Inokuchi K, Soejima K, Matsusaka T, Okamura T. Growth patterns and prognosis in early gastric carcinoma. Superficially spreading and penetrating growth types. Cancer 1985;51:320-6.

21. Mochizuki F, Fujii M, Kasakura Y, Imai S, Yamagata T, Hasegawa T. Clinicopathological analysis of recurrence in early gastric cancer (in Japanese). J Nihon Univ Med Assoc 1997;56:1520.

22. Sato K, Kumagai K, Shirasawa K, Tsujimoto Y, Watanabe Y, Kidokoro $\mathrm{T}$, et al. A case of elevated early gastric carcinoma occupied the upper third of the stomach (in Japanese). J Jpn Surg Assoc 1986;47:623-7.

23. Kitamura K, Yamaguchi T, Okamoto K, Nishida T, Takahashi T. Superficial spreading type of early gastric cancer. Br J Cancer 1996;74:1834-7.

24. Iwanaga T, Taniguchi H, Wada A, Kosaki G. Histopathological study on superficial spreading type of early gastric cancer (in Japanese). Jpn J Cancer Clin 1967;13:338-48.

25. Yasui A. Histopathological feature of superficial spreading type of early gastric cancer. A symposium report from the 29th Annual Meeting of the Japanese Cancer Association (in Japanese). 1970;95-101.

26. Oiwa H, Maehara Y, Ohno S, Sakaguchi Y, Ichiyoshi Y, Sugimachi K. Growth pattern and p 53 overexpression in patients with early gastric cancer. Cancer 1995;6:1454-9.

27. Maruyama K, Okabayashi K, Kinoshita T. Progress in gastric cancer surgery in Japan and its limits of radicality. World J Surg 1987;11:418-25.

28. Takahara O, Takahara H, Yamashita I, Noguchi K. A case of so-called superficial spreading type early gastric cancer (grouped IIa and IIb-like IIc) (in Japanese). Stomach and Intestine 1976;11:641-6.

29. Kasakura Y, Karube H, Tsuneda Y, Ishii Y, Murayama I, Sato H. A case of superficial wide spreading type of early gastric cancer (in Japanese). Geka Shinryo (Surgical Treatment) 1993;35:75560.

30. Kitamura K, Yamaguchi T, Okamoto K, Takahashi T, Sugihara H. Superficial spreading type of early gastric lymphomas. Anticancer Res 1995;15:2829-34.

31. Kitamura K, Yamaguchi T, Okamoto K, Ichikawa D, Hoshino M, Tanibuchi H. Early gastric lymphoma. A clinicopathologic study of ten patients, literature review, and comparison with early gastric adenocarcinoma. Cancer 1996;77:850-7.

32. Sekiguchi R, Asada Y, Hayashi K, Togoh A, Ijima N, Fujimoto T, et al. Clinicopathological evaluation of endoscopic diagnosis in superficial spreading type of early gastric cancer (in Japanese). Progr Dig Endosc 1988;33:138-42.

33. Yoshimura Y, Yasutake K, Imamura Y, Sashikata T, Oimomi M. Endoscopic studies on the superficial spreading type of early gastric cancer. Kobe J Med Sci 1989;35:29-38.

34. Takeshita K, Mori S, Yamauchi H, Gen U, Senyo G, Hiraide H, et al. Surgical consideration toward the superficial spreading type of early gastric cancer (in Japanese). Progr Dig Endos 1976;8:122-4.

35. Richart RM. A clinical staining test for the in vivo delineation of dysplasia and carcinoma in site. Am J Obstet Gynecol 1963; 86:703-12. 